\title{
A Mathematical Model for Schistosomiasis Japonicum with Harmless Delay
}

\author{
Huahua Cao, Shujing Gao*, Xiangyu Zhang, Youquan Luo \\ College of Mathematics and Computer Science, Gannan Normal University, Ganzhou, China \\ Email: 870577346@qq.com, gaosjmath@126.com, xyzhang5@163.com
}

Received 12 August 2014; revised 28 August 2014; accepted 16 September 2014

Copyright (C) 2014 by authors and Scientific Research Publishing Inc.

This work is licensed under the Creative Commons Attribution International License (CC BY). http://creativecommons.org/licenses/by/4.0/

c) (i) Open Access

\begin{abstract}
From the lifecycle of schistosome, the phenomenon of time delay is widespread. In this paper, a two-dimensional system is studied that incorporates two time delays which are the incubation period of human and snail, respectively. Our purpose is to demonstrate that the time delays are harmless for stability of equilibria of the system. Further, sufficient conditions of stability of equilibria are obtained.
\end{abstract}

\section{Keywords}

Stability, Schistosomiasis Japonicum, Time Delay

\section{Introduction}

Mathematical models ([1]-[7], etc.) have been used to study the transmission and control of schistosomiasis since the first model that has been given by MacDonald in [8]. MacDonald's model consists of two differential equations in two state variables that correspond to average parasite burden in the definitive hosts and the prevalence of infection in snails. DAS et al. [5] added a layer of biological realism to these early models to study the delay effect on schistosomiasis transmission with control measures. The model is given by

$$
\left\{\begin{array}{l}
\frac{\mathrm{d} x}{\mathrm{~d} t}=\alpha \sigma y(t)-\gamma x-C, \\
\frac{\mathrm{d} y}{\mathrm{~d} t}=\beta x(t-\tau)\left(1-\frac{y}{N}\right)-\delta y-H,
\end{array}\right.
$$

where $x$ is the current number of egg laying schistosomes in the human host population and $y$ is the current

\footnotetext{
*Corresponding author.
} 
number of infected snails in the environment. Here, $\sigma$ is the human population density per unit accessible water area; $\alpha$ is the multiplication rate due to the infected snail population; $\gamma$ and $\delta$ are the intrinsic death rates of two populations $x$ and $y$ respectively; $\beta$ is the simple contact rate; $C$ is the constant decay rate due to chemotherapy; and $H$ is the constant decay rate by predation or harvesting. Further $\tau$ is the incubation period for becoming $y$ to be infectious. For simplicity, it is assumed that $N$ is the constant total population of snails.

In [5], for the sake of mathematical simplicity, they assumed the development of schistosoma is instantaneous. In fact, the developmental time of schistosome is not short. Under normal circumstances, the transit time from parasite eggs to miracidia to infect snail is about 21 days, cercariae are produced about 44 - 159 days after the miracidium penetration in snail hosts. In this paper, we also assume that $C$ and $H$ are the proportions of chemotherapy and predation or harvesting, respectively. Based on the above description, a schistosomiasis model with two time delays is proposed:

$$
\left\{\begin{array}{l}
\frac{\mathrm{d} x}{\mathrm{~d} t}=\alpha \sigma y\left(t-\tau_{1}\right)-(\gamma+C) x \\
\frac{\mathrm{d} y}{\mathrm{~d} t}=\beta x\left(t-\tau_{2}\right)\left(1-\frac{y}{N}\right)-(\delta+H) y,
\end{array}\right.
$$

where $\tau_{1}$ is the incubation period for becoming infected human host population and $\tau_{2}$ is the transit time from parasite eggs to miracidia to infect snail. We assume that all parameters are positive.

From biological view, we assume that system (2) holds for the time $t>0$ with given nonnegative initial conditions:

$$
x(\theta)=\phi(\theta) \geq 0, \quad y(\theta)=\psi(\theta) \geq 0, \quad \theta \in[-\tau, 0), \quad \phi(0)>0, \quad \psi(0)>0,
$$

where $(\phi(\theta), \psi(\theta)) \in C\left([-\tau, 0], \mathbb{R}_{+0}^{2}\right)$, the Banach space of continuous functions mapping the interval [- $\left.\tau, 0\right]$ into $\mathbb{R}_{+0}^{2}$, where $\mathbb{R}_{+0}^{2}=\left\{\left(x_{1}, x_{2}\right): x_{i} \geq 0, i=1,2\right\}$.

In the following, we focus on dynamics of system (2) in a nonnegative cone

$$
D=\left\{(x, y) \in R^{2}: x \geq 0, y \geq 0\right\}
$$

It is well known by the fundamental theory of functional differential equations [9] that system (2) has a unique solution $(x(t), y(t))$ satisfying initial conditions (3). It is easy to show that all solutions of system (2) corresponding to initial conditions (3) are defined on $[0,+\infty)$ and remain positive for all $t \geq 0$.

The remainder of the paper is organized as follows. In the next section, the stability of the disease-free equilibrium of system (2) is obtained. In Section 3, we investigate the stability of the endemic equilibrium. Some dynamical behaviors are given by numerical simulations in Section 4. This paper is ended with a brief discussion.

\section{Stability Analysis of the Disease-Free Equilibrium}

In this section, the stability of the disease-free equilibrium of system (2) is investigated.

Using standard methods, it is easy to see that the disease-free equilibrium $E_{0}=(0,0)$ always exists.

Define the basic reproductive number by

$$
R_{0}=\frac{\alpha \beta \sigma}{(\gamma+C)(\delta+H)} .
$$

Then for system (2), it is easy to obtain the following result:

(i) If $R_{0}<1$, system (2) has a unique disease-free equilibrium $E_{0}$;

(ii) If $R_{0}>1$, system (1.2) has two equilibria, the disease-free equilibrium $E_{0}$ and the unique endemic equilibrium $E^{*}\left(x^{*}, y^{*}\right)$, where

$$
\begin{aligned}
& x^{*}=\frac{N}{\beta(\gamma+C)}[\alpha \beta \sigma-(\delta+H)(\gamma+C)], \\
& y^{*}=\frac{N}{\alpha \beta \sigma}[\alpha \beta \sigma-(\delta+H)(\gamma+C)] .
\end{aligned}
$$


In the following, we study the global stability of the disease-free equilibrium $E_{0}$ of system (2).

Theorem 2.1. If $R_{0}<1$, the disease-free equilibrium $E_{0}$ of system (2) is locally asymptotically stable.

Proof. First, according to [9], the Jacobian matrix at $E_{0}$ of system (2) can be written as

$$
J(0,0)=\left(\begin{array}{cc}
-(\gamma+C) & \alpha \sigma \mathrm{e}^{-\lambda \tau_{1}} \\
\beta \mathrm{e}^{-\lambda \tau_{2}} & -(\delta+H)
\end{array}\right)
$$

Then the characteristic equation of system (2) at $E_{0}$

$$
\lambda^{2}+p \lambda+q-m \mathrm{e}^{-\lambda \tau}=0,
$$

where $p=\delta+H+\gamma+C>0, \quad q=(\gamma+C)(\delta+H)>0, \quad m=\alpha \beta \sigma>0, \quad \tau=\tau_{1}+\tau_{2}$.

When $\tau=0$, (5) becomes into

$$
\lambda^{2}+p \lambda+q-m=0
$$

If $q>m$, the roots of the equation (6) have negative real parts. Note that $q>m$ is equivalent to $R_{0}<1$. Therefore, if $R_{0}<1$ and $\tau=0, E_{0}$ is locally asymptotically stable.

Assume that there exists a $\tau_{0}>0$ such that (5) has pure imaginary roots $\lambda= \pm i \omega(\omega>0)$. Then we have from (5) that

$$
-\omega^{2}+q+p \omega i-m(\cos \omega \tau-i \sin \omega \tau)=0 .
$$

Separating real and image parts:

$$
\left\{\begin{array}{l}
-\omega^{2}+q=m \cos \omega \tau, \\
-p \omega=m \sin \omega \tau .
\end{array}\right.
$$

Adding up the squares of both equations, we obtain that

$$
\omega^{4}+\left(p^{2}-2 q\right) \omega^{2}+q^{2}-m^{2}=0
$$

Note that

$$
p^{2}=[(\delta+H)+(\gamma+C)]^{2}=(\delta+H)^{2}+(\gamma+C)^{2}+2(\delta+H)(\gamma+C) \geq 4(\delta+H)(\gamma+C)=4 q
$$

and $q>m$ Thus, $q^{2}>m^{2}$, which implies that (7) has no positive roots, i.e., $\tau_{0}$ does not exist. This yields that all roots of (5) have negative real parts if $R_{0}<1$.

Next, the global stability of the disease-free equilibrium of system (2) is analyzed. And the strategy of proof is to use Lyapunov functionals and the LaSalle invariance principle.

Theorem 2.2. If $R_{0}<1$, the disease-free equilibrium $E_{0}$ is globally asymptotically stable in $D$ for all $\tau>0$.

Proof. Let $(x(t), y(t))$ be any positive solution of system (2) with initial conditions (3).

Define

$$
V_{11}(t)=c_{1} x+c_{2} y
$$

where $c_{1}=\beta, c_{2}=\gamma+C$.

Calculating the derivative of $V_{11}(t)$ along positive solutions of system (2), it follows that

$$
\begin{aligned}
\dot{V}_{11}(y) & =c_{1}\left[\alpha \sigma y\left(t-\tau_{1}\right)-(\gamma+C) x\right]+c_{2}\left[\beta x\left(t-\tau_{2}\right)\left(1-\frac{y}{N}\right)-(\delta+H) y\right] \\
& =c_{1} \alpha \sigma y\left(t-\tau_{1}\right)-c_{2}(\delta+H) y+c_{2} \beta x\left(t-\tau_{2}\right)-c_{1}(\gamma+C) x-c_{2} \beta x\left(t-\tau_{2}\right) \frac{y}{N} .
\end{aligned}
$$

Define 


$$
V_{12}(t)=V_{11}(t)+c_{1} \alpha \sigma \int_{t-\tau_{1}}^{t} y(s) \mathrm{d} s
$$

We derive from (8) and (9) that

$$
\begin{aligned}
\dot{V}_{12}(t) & =\dot{V}_{11}(t)+c_{1} \alpha \sigma\left[y(t)-y\left(t-\tau_{1}\right)\right] \\
& =\left[c_{1} \alpha \sigma-c_{2}(\delta+H)\right] y+c_{2} \beta x\left(t-\tau_{2}\right)-c_{1}(\gamma+C) x-c_{2} \beta x\left(t-\tau_{2}\right) \frac{y}{N} .
\end{aligned}
$$

Define

$$
V_{1}(t)=V_{12}(t)+c_{2} \beta \int_{t-\tau_{2}}^{t} x(s) \mathrm{d} s
$$

It follows from (10) and (11) that

$$
\dot{V}_{1}(t)=\dot{V}_{12}(t)+c_{2} \beta\left[x(t)-x\left(t-\tau_{2}\right)\right]=\left[c_{1} \alpha \sigma-c_{2}(\delta+H)\right] y+\left[c_{2} \beta-c_{1}(\gamma+C)\right] x-c_{2} \beta x\left(t-\tau_{2}\right) \frac{y}{N}
$$

On substituting $c_{1}=\beta$ and $c_{2}=(\gamma+C)$ into (12), we obtain that

$$
\dot{V}_{1}(t)=[\alpha \beta \sigma-(\gamma+C)(\delta+H)] y-\frac{\beta(\gamma+C)}{N} x\left(t-\tau_{2}\right) y .
$$

If $R_{0}<1$, that is $\alpha \beta \sigma-(\gamma+C)(\delta+H)<0$, it then follows from (13) that $\dot{V}_{1}(t) \leq 0$. By Theorem 5.3.1 in [9], solutions limit to $M$, the largest invariant subset of $\left\{\dot{V}_{1}(t)=0\right\}$. Clearly, we see from (13) that $\dot{V}_{1}(t)=0$ if and only if $y=0$. Noting that $M$ is invariant, for each element in $M$, we have $y=0$. It therefore follows from the second equation of system (2) that

$$
0=\dot{y}(t)=\beta x\left(t-\tau_{2}\right),
$$

which yields $x(t)=0$. Hence, $\dot{V}_{1}(t)=0$ if and only if $(x, y)=(0,0)$. Accordingly, the global asymptotic stability of $E_{0}$ follows from LaSalle's invariance principle.

\section{Stability Analysis of the Endemic Equilibrium}

It is obtained that the endemic equilibrium $E^{*}$ of system (2) is local stable in this section. Further, the global stability of $E^{*}$ is shown if $\tau=0$.

Similar to the proof of Theorem 2.1, the following result is obtained.

Theorem 3.1. If $R_{0}>1$, the endemic equilibrium $E^{*}$ of system (2) is locally asymptotically stable.

Proof. First, according to [9], the Jacobian matrix at $E^{*}$ can be written as

$$
J\left(x^{*}, y^{*}\right)=\left(\begin{array}{cc}
-(\gamma+C) & \alpha \sigma \mathrm{e}^{-\lambda \tau_{1}} \\
\frac{(\delta+H)(\gamma+C)}{\alpha \sigma} \mathrm{e}^{-\lambda \tau_{2}} & -\frac{\alpha \beta \sigma}{\gamma+C}
\end{array}\right)
$$

Then the characteristic equation of system (2) at $E^{*}$ :

$$
\lambda^{2}+\left[(\gamma+C)+\frac{\alpha \beta \sigma}{\gamma+C}\right] \lambda+\alpha \beta \sigma-(\delta+H)(\gamma+C) \mathrm{e}^{-\lambda \tau}=0,
$$

where $\tau=\tau_{1}+\tau_{2}$.

When $\tau=0$, (15) becomes into

$$
\lambda^{2}+\left[(\gamma+C)+\frac{\alpha \beta \sigma}{\gamma+C}\right] \lambda+\alpha \beta \sigma-(\delta+H)(\gamma+C)=0,
$$

If $R_{0}>1$, then $\alpha \beta \sigma-(\delta+H)(\gamma+C)>0$. It is shown that all the roots of the Equation (16) have negative 
real parts, suggesting $E^{*}$ is locally asymptotically stable.

Assume that there exists a $\tau_{0}^{\prime}>0$ such that (15) has pure imaginary roots $\lambda= \pm i \omega(\omega>0)$. Then we have from (15) that

$$
-\omega^{2}+\left[(\gamma+C)+\frac{\alpha \beta \sigma}{\gamma+C}\right] \omega i+\alpha \beta \sigma-(\delta+H)(\gamma+C)(\cos \omega \tau-i \sin \omega \tau)=0 .
$$

Separating real and image parts:

$$
\left\{\begin{array}{l}
-\omega^{2}+\alpha \beta \sigma=(\delta+H)(\gamma+C) \cos \omega \tau \\
{\left[(\gamma+C)+\frac{\alpha \beta \sigma}{\gamma+C}\right] \omega=-(\delta+H)(\gamma+C) \sin \omega \tau .}
\end{array}\right.
$$

Adding up the squares of both equations, we obtain that

$$
\omega^{4}+\left[(\gamma+C)^{2}+\frac{\alpha^{2} \beta^{2} \sigma^{2}}{(\gamma+C)^{2}}\right] \omega^{2}+\alpha^{2} \beta^{2} \sigma^{2}-(\delta+H)^{2}(\gamma+C)^{2}=0 .
$$

We know that $\alpha^{2} \beta^{2} \sigma^{2}-(\delta+H)^{2}(\gamma+C)^{2}>0$ if $R_{0}>1$, so (17) has no positive roots, i.e., $\tau_{0}^{\prime}$ does not exist. This yields that all roots of (17) have negative real parts if $R_{0}>1$.

Now, we are interested in the global stability of $E^{*}$. Then its global stability is investigated by means of Bendixson theorem.

Theorem 3.2. If $R_{0}>1$, the endemic equilibrium $E^{*}$ is globally asymptotically stable in $D$ when $\tau=0$.

Proof. It is easy to check that equilibrium $E_{0}$ of system (2) is unstable if $R_{0}>1$. By the above discussion, we know that equilibrium $E^{*}$ is locally stable if $R_{0}>1$ and all solutions of system (2) are ultimately bounded in $D$. To prove the second assertion, we only prove that system (2) has not periodic orbits in the interior of $D$ if $R_{0}>1$.

When $\tau=0$,

$$
\left\{\begin{array}{l}
\frac{\mathrm{d} x}{\mathrm{~d} t}=\alpha \sigma y-(\gamma+C) x \triangleq P, \\
\frac{\mathrm{d} y}{\mathrm{~d} t}=\beta x\left(1-\frac{y}{N}\right)-(\delta+H) y \triangleq Q .
\end{array}\right.
$$

It follows that

$$
\frac{\partial P}{\partial x}+\frac{\partial Q}{\partial y}=-(\gamma+C)-(\delta+H)-\frac{\beta x}{N}<0
$$

which leads to the nonexistence of periodic orbits by Bendixson theorem, therefore, $E^{*}$ is globally asymptotically stable.

\section{Numerical Simulations}

It is reported that cercariae are produced about 44 - 159 days after the miracidium penetration in snails. And the time from parasite eggs to miracidia to infect snail is about 21 days. Therefore, we choose $\tau_{1}=45$ and $\tau_{2}=21$ in this paper. Further, in this section, we perform some numerical simulations and sensitivity analysis using the following value of parameters: $\alpha=0.55, \beta=0.48, \sigma=0.059, C=0.15, H=0.10, \delta=0.012$, $\gamma_{1}=0.0055, \quad N=10^{6}$.

Thus, we can obtain $R_{0}=0.894<1$, the disease-free equilibrium $(0,0)$ is asymptotically stable (Figure 1(a)). When $H=0.08$, the value of other parameters is fixed, we can obtain $R_{0}=1.089>1$ and the unique endemic equilibrium is asymptotically stable (Figure 1(b)). In addition, fixing $H$ in simulations, we find that the number of parasite eggs and infectious snails increases as $C$ decreases, respectively (Figure 2).

From the above theorems, we know that the two time delays are harmless. According to the expression of $R_{0}$, 

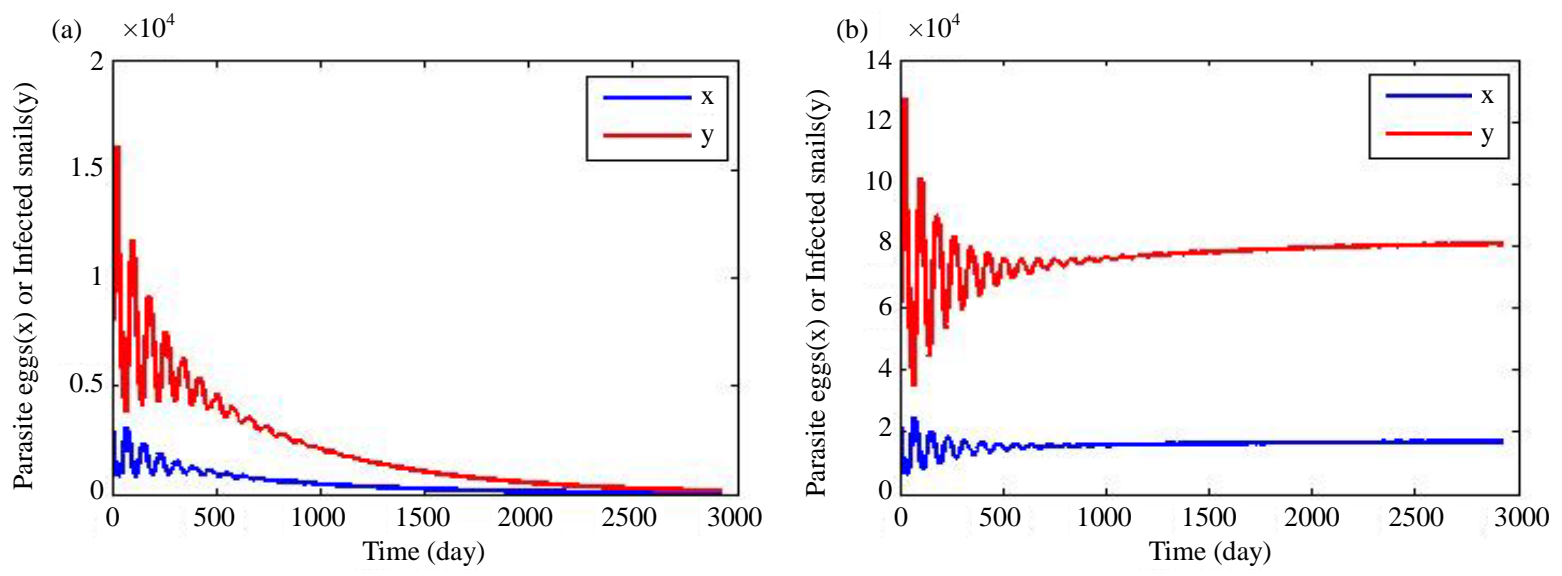

Figure 1. The figure (a) shows that a numerical solution of system (2) tends to the disease-free equilibrium as time tends to infinity, where $R_{0}=0.894$. The figure (b) illustrates that a numerical solution of system (2) tends to the endemic equilibrium as time tends to infinity, where $R_{0}=1.089$.
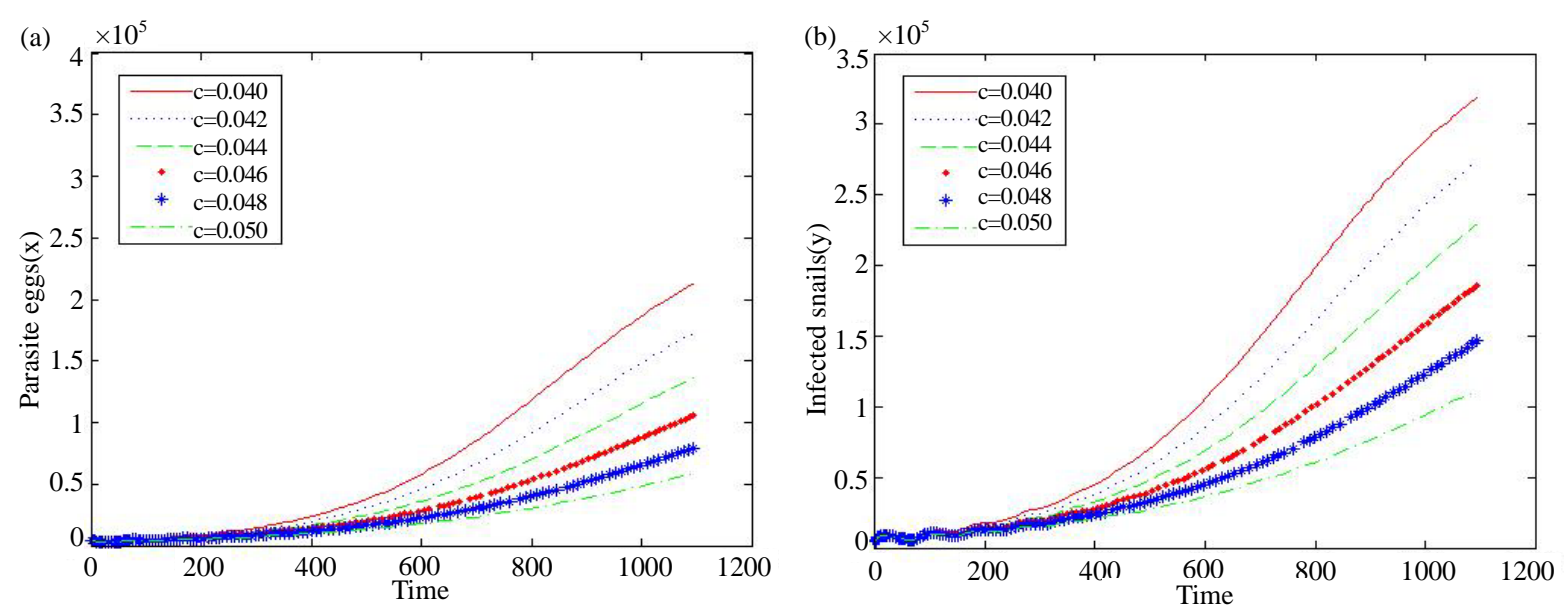

Figure 2. Simulation results: $C=0.040,0.042,0.044,0.046,0.048,0.050$ from top to base, respectively. We can find that the smaller of values of $C$, the higher of values of parasite eggs and infected snails.

the impact of $C$ and $H$ on schistosomiasis transmission is discussed. Fixing $H=0.10$, we can see that when $C \in[0,0.13]$, the endemic equilibrium exists and is stable, when $C>0.13$, the endemic equilibrium doesn't exist. But the disease-free equilibrium is stable (Figure 3(a)). Analogously, fixing $C=0.15$, from Figure 3(b), it is obvious that the disease-free equilibrium is stable when $H>0.08$.

From the formula of the basic reproductive number, we know that the basic reproductive number is a decrease function of the rates of chemotherapy and predation or harvesting. This means chemotherapy and predation or harvesting can influence the system.

However, to find out the most influential control measure, we need sensitivity analysis. Now we carry out the sensitivity analysis by calculating the derivation of $R_{0}$ on $C$ and $H$. The derivation is respectively

$$
\begin{aligned}
& \frac{\partial R_{0}}{\partial C}=-\frac{\alpha \beta \sigma}{(\gamma+C)^{2}(\delta+H)}, \\
& \frac{\partial R_{0}}{\partial H}=-\frac{\alpha \beta \sigma}{(\gamma+C)(\delta+H)^{2}} .
\end{aligned}
$$

From Figure 4(a), we can see that when $C<0.2, R_{0}$ decreases rapidly with the increase of $C$, the decline of $R_{0}$ is not obvious. Similarly, $R_{0}$ decreases rapidly with the increase of $H$ when $H<0.25$ (Figure 4(b)). 

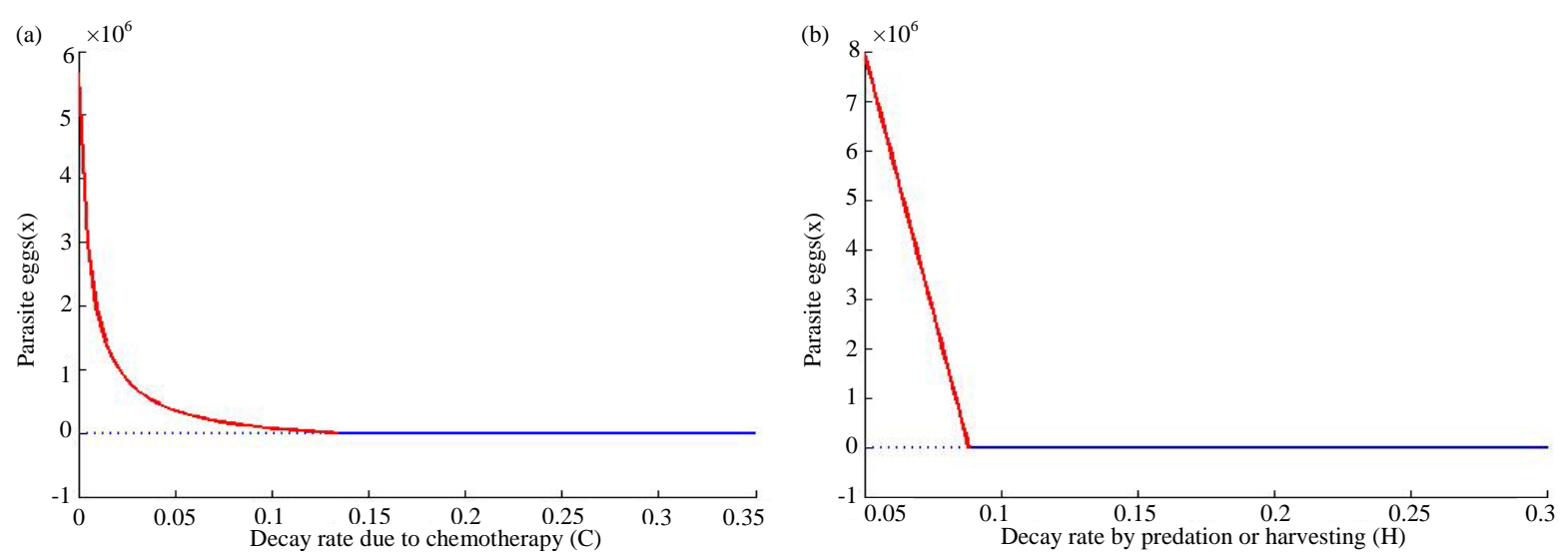

Figure 3. Forward bifurcation diagrams for the parasite eggs population.
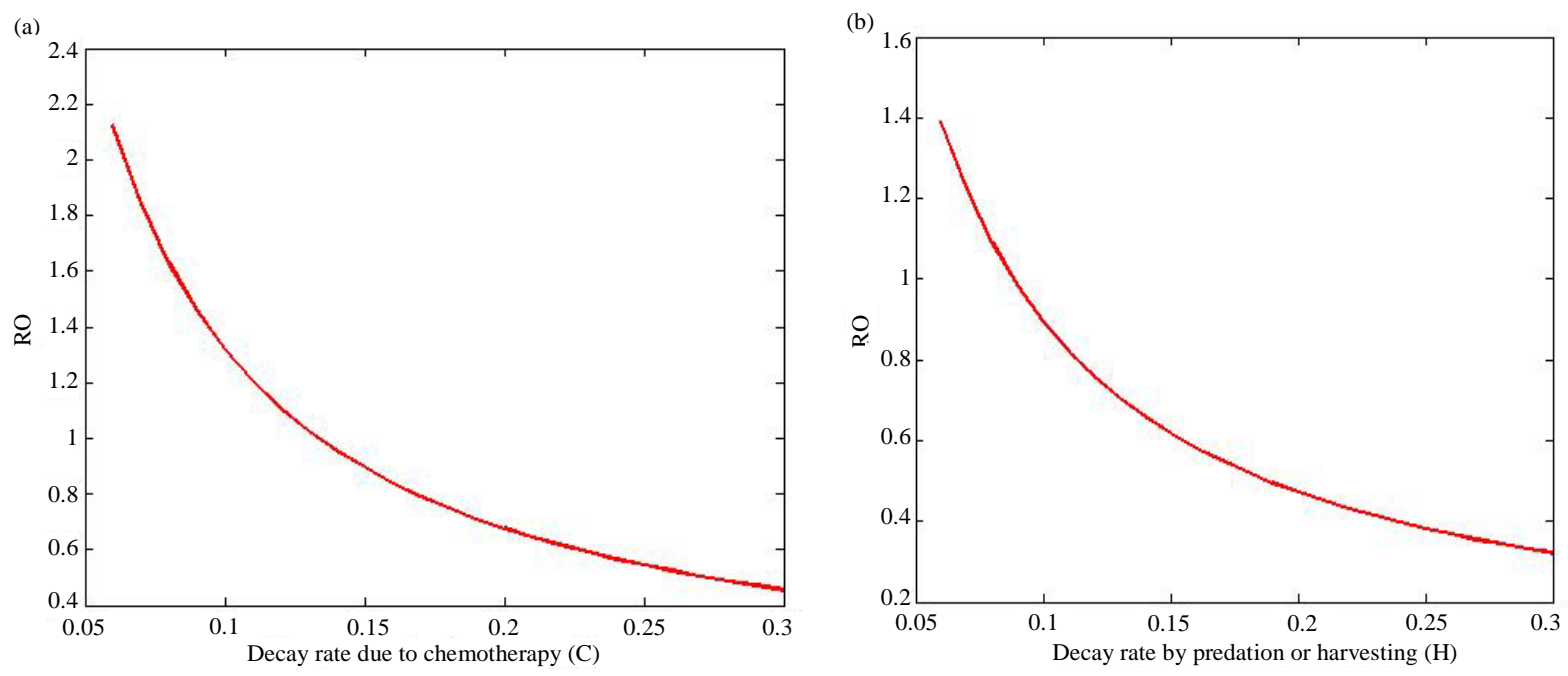

Figure 4. Sensitivity analysis of $R_{0}$ on $C$ and $H$, respectively.

In brief, the basic reproductive number $R_{0}$ is more sensitive when $C$ and $H$ are small.

By sensitivity analysis of the basic reproductive number on the rates of chemotherapy and predation or harvesting, we know that the basic reproductive number is a decrease function of the rates of chemotherapy and predation or harvesting. In numerical simulations, we also find that the smaller of values of the rate of chemotherapy, the more sensitive of the basic reproductive number $R_{0}$.

Although the two time delays are harmless, all of these results imply that the rates of chemotherapy and predation or harvesting can influence the dynamic behaviors. Furthermore, to reduce the prevalence of schistosomiasis infection, to some extent, increasing the rate of predation or harvesting by some measures could achieve better results than increasing the rate of chemotherapy.

\section{Conclusions}

In this paper, we propose a system of delayed differential equations for schistosomiasis japonicum transmission and obtain sufficient conditions for the existence and local stability of equilibria. Further, global asymptotic stability of the disease-free equilibrium is also studied by constructing suitable Lyapunov functions. When $R_{0}<1$, the disease-free equilibrium is globally asymptotically stable (Figure 5(a)); when $R_{0}>1$, the endemic-free equilibrium is locally asymptotically stable and globally asymptotically stable if $\tau=\tau_{1}+\tau_{2}=0$. Thus, $R_{0}$ plays an important part in controlling schistosomiasis.

Finally, we guess that the endemic equilibrium should be global asymptotic stable when $\tau>0$. And this guess is verified by numerical simulations (Figure 5(b)). This issue will be addressed in future studies. 

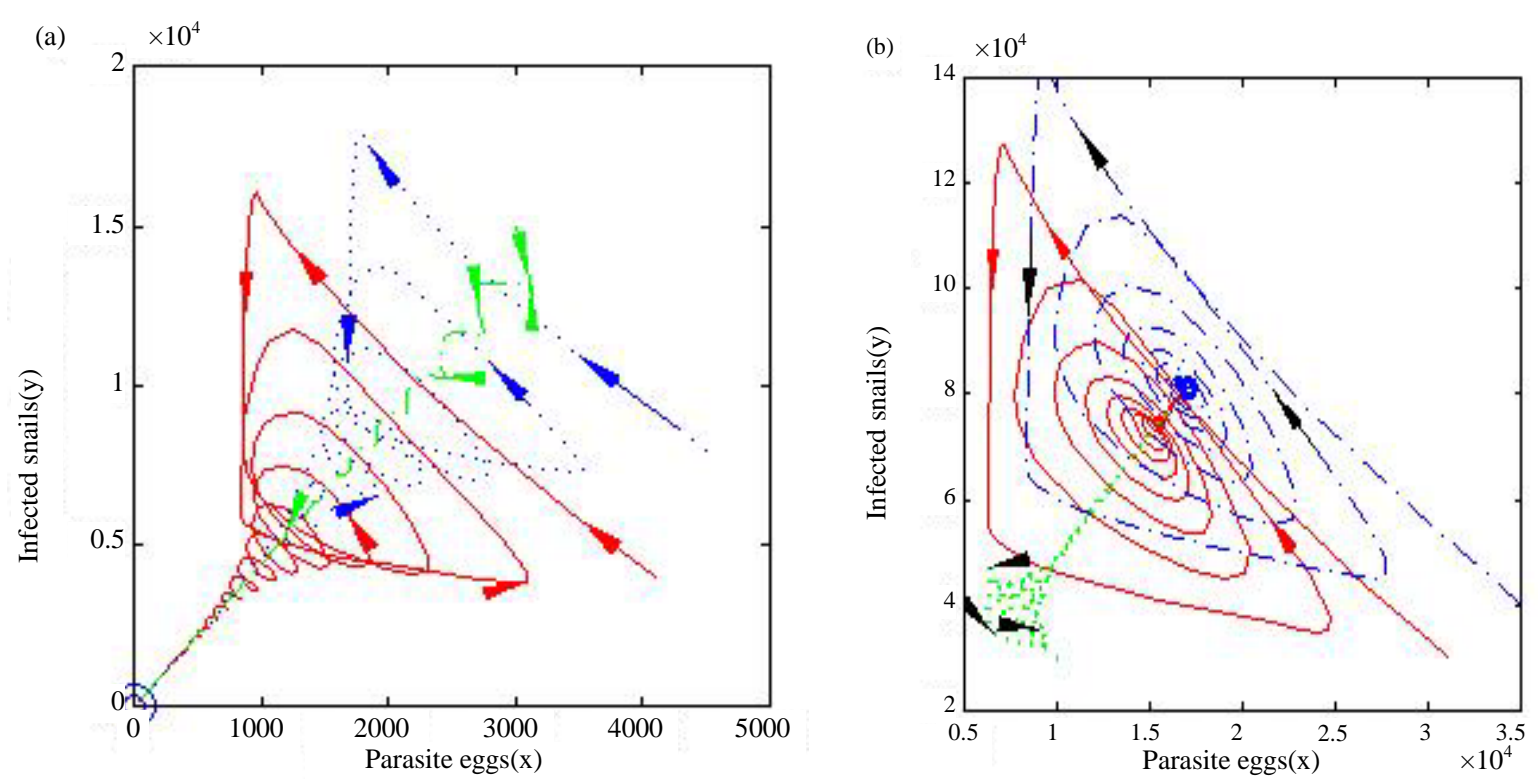

Figure 5. Phase diagrams: (a) $R_{0}=0.894$; (b) $R_{0}=1.089$.

\section{Acknowledgements}

The research has been partially supported by The Natural Science Foundation of China (No. 11261004), China Postdoctoral Science Foundation funded project (No. 2012M510039), the National Key Technologies R \& D Program of China (2009BAI78B01, 2009BAI78B02) and the Natural Science Foundation of Jiangxi (20114 BAB201013, 20122BAB211010).

\section{References}

[1] Anderson, R.M. and May, R.M. (1985) Helminth Infections of Humans Mathematical Models, Population Dynamics, and Control. Advances in Parasitology, 24, 1-101. http://dx.doi.org/10.1016/S0065-308X(08)60561-8

[2] Barbour, A.D. (1996) Modeling the Transmission of Schistosomiasis: An Introductory View. The American Journal of Tropical Medicine and Hygiene, 55, 135-143.

[3] Woolhouse, M.E. (1991) On the Application of Mathematical Models of Schistosome Transmission Dynamics. I. Natural Transmission. Acta Tropica, 49, 241-270. http://dx.doi.org/10.1016/0001-706X(91)90077-W

[4] Yang, H.M. (2003) Comparison between Schistosomiasis Transmission Modeling Considering Acquired Immunity and Age-Structured Contact Pattern with Infested Water. Mathematical Biosciences, 184, 1-26. http://dx.doi.org/10.1016/S0025-5564(03)00045-2

[5] Das, P., Mukherjee, D. and Sarkar, A.K. (2006) A Study of Schistosome Transmission Dynamics and Its Control. Journal of Biological Systems, 14, 295-302. http://dx.doi.org/10.1142/S0218339006001799

[6] Chiyaka, E.T. and Garira, W. (2009) Mathematical Analysis of the Transmission Dynamics of Schistosomiasis in the Human-Snail Host. Journal of Biological Systems, 17, 397-423. http://dx.doi.org/10.1142/S0218339009002910

[7] Qi, L.X. and Cui, J. (2012) Modeling the Schistosomiasis on the Islets in Nanjing. International Journal of Biomathematics, 5, 189-205.

[8] Macdonald, G. (1965) The Dynamics of Helminth Infections, with Special Reference to Schistosomes. Transactions of the Royal Society of Tropical Medicine and Hygiene, 59, 489-506. http://dx.doi.org/10.1016/0035-9203(65)90152-5

[9] Hale, J.K. (1976) Theory of Functional Differential Equations. Springer, New York. 
Scientific Research Publishing (SCIRP) is one of the largest Open Access journal publishers. It is currently publishing more than 200 open access, online, peer-reviewed journals covering a wide range of academic disciplines. SCIRP serves the worldwide academic communities and contributes to the progress and application of science with its publication.

Other selected journals from SCIRP are listed as below. Submit your manuscript to us via either submit@scirp.org or Online Submission Portal.
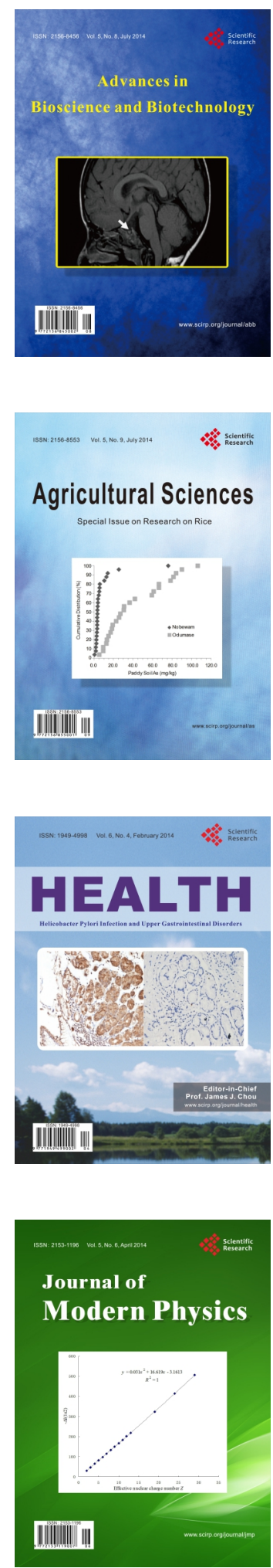
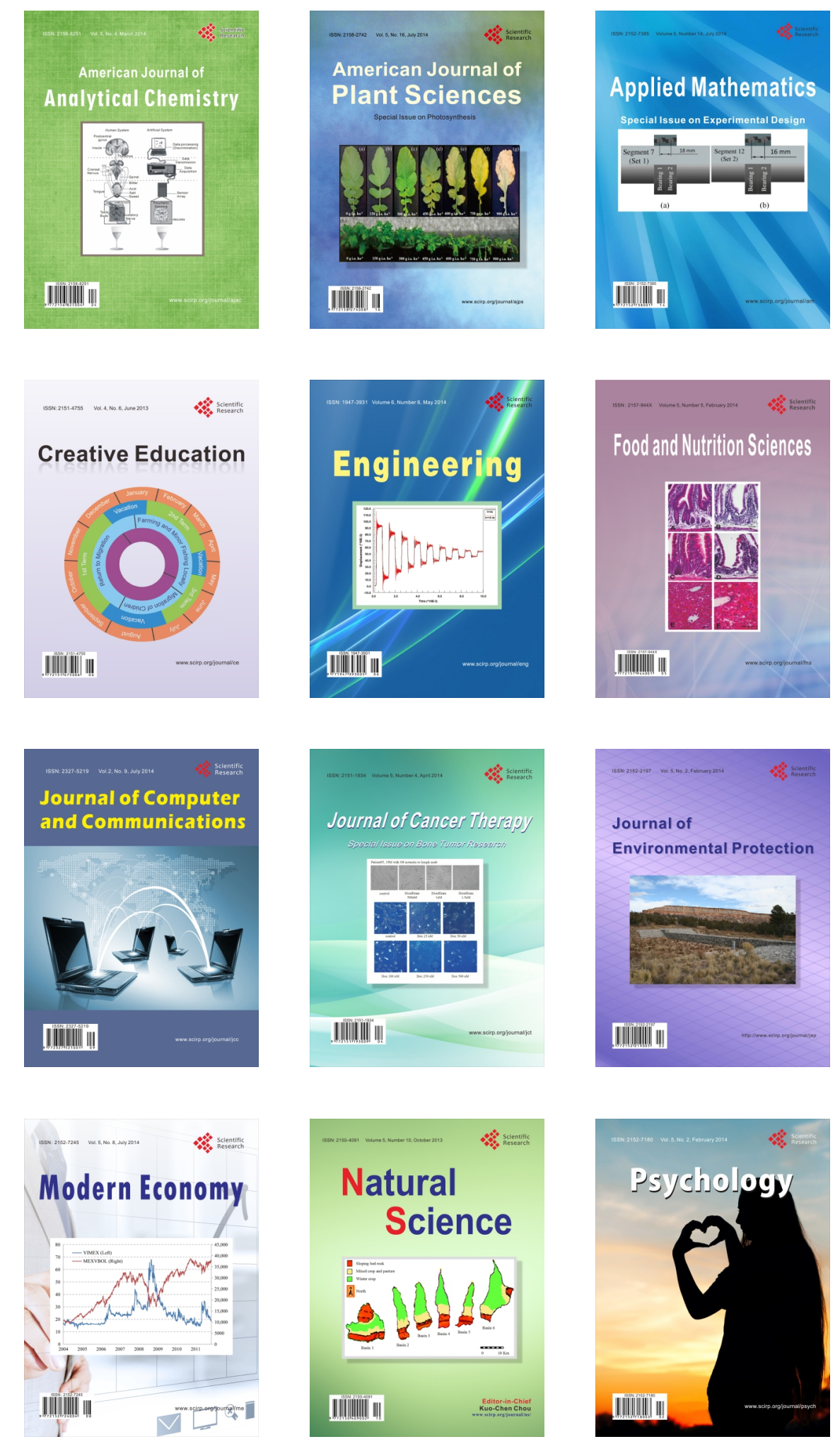